\title{
RESISTÊNCIA DE SOJA A INSETOS. V. PREFERÊNCIA PARA ALIMENTAÇÃO DE ADULTOS DE DIPHAULACA VIRIDIPENNIS CLARK, 1865, EM CULTIVARES E LINHAGENS (1)
}

\author{
ANDRÉ LUIZ LOURENÇĀO $(2,4)$ \\ e MANOEL ALBINO COELHO DE MIRANDA $(3,4)$
}

\begin{abstract}
RESUMO
Durante o ano agrícola 1983/84, em condiçōes de casa de vegetação, estudou-se em quatro experimentos a preferência para alimentação de adultos de Diphaulaca viridipennis Clark, 1865,em dois cultivares e nove linhagens de soja. Três experimentos constituíram-se em testes tipo livre chance de escolha: no primeiro, a linhagem PI 227687 apresentou perda de área foliar menor que os demais tratamentos ('Santa Rosa', 'IAC-8', PI 171451 e PI 229358). No segundo e no terceiro experimento, em que se manteve PI 227687 e 'Santa Rosa' como, respectivamente, padrāo de resistência e suscetibilidade, as linhagens comparadas (PI 274453, IAC 78-2318, IAC 79-1823, IAC 80-1177, IAC 80-596-2 e D 72-9601-1) exibiram danos foliares intermediários entre esses padrōes. $O$ quarto experimento constou de um teste sem chance de escolha envolvendo o tratamento menos danificado nos três experimentos iniciais, PI 227687, e o mais suscetível, 'Santa Rosa'; as porcentagens médias de área foliar comida não diferiram entre os dois materiais, indicando que a resistência observada na linhagem PI 227687 não se mantém sob condiçôes de obrigatoriedade de alimentação por adultos de $D$. viridipennis.
\end{abstract}

Termos de indexação: resistência a insetos, preferência para alimentação, soja, Glycine $\max ($ L.) Merrill, Diphaulaca viridipennis Clark, 1865, área foliar comida.

\footnotetext{
(1) Recebido para publicação em 7 de novembro de 1984. (SP).

(2) Seção de Entomologia Fitotécnica, Instituto Agronômico (IAC), Caixa Postal 28, 13001 Campinas

(3) Seção de Leguminosas, IAC.

(4) Com bolsa de suplementação do $\mathrm{CNPq}$.
} 


\section{INTRODUÇÃO}

Diversos coleópteros da família Chrysomelidae têm sido observados em lavouras de soja no Estado de São Paulo, sendo os mais comuns Diabrotica speciosa (Germar) (MASSARIOL et alii, 1979; RODINI \& GRAZIA, 1979, e WILLIAMS et alii, 1973), Cerotoma arcuata (Olivier) (RODINI \& GRAZIA, 1979, e ROSSETTO et alii, 1981) e Colaspis sp., possivelmente C. occidentalis (L.) (REZENDE et alii, 1980; ROSSETTO \& NAGAl, 1980, e WILLIAMS et alii, 1973).

Durante a safra 1983/84, um crisomelídeo da subfamilia Alticinae, com cerca de $6 \mathrm{~mm}$ de comprimento, pronoto alaranjado e élitros de coloração azul-metálica, foi obsenvado infestando intensamente canteiros de soja linhagem IAC 77-530, no Centro Experimental de Campinas. Ao lado dos canteiros, verificou-se também a presença e alimentaçāo do inseto em cudzu (Pueraria thumbergiana Benth.), caracterizando-a como outra planta-alimento favorável. Além desse campo altamente infestado, tal espécie de besourinho ocorreu nessa safra de forma generalizada em todos os campos experimentais de soja da Seção de Entomologia em Campinas. Exemplares do inseto foram coletados, montados e catalogados na coleção da Seção de Entomologia Fitotécnica do Instituto Agronômico de Campinas, sob n- 6804, e identificados pelo Dr. R.E. White (Insect Identification and Beneficial Insect Introduction Institute, Beltsville, EUA), como Diphaulaca sp. Todavia, a ocorrência em soja na região de Dourados, MS, de um inseto bastante semelhante aos exemplares de Diphaulaca, denominado Chlamophora sp. (DEGASPARI \& GOMEZ, 1979) motivou a remessa do material de Dourados $\left({ }^{5}\right.$ ), catalogado na coleção supramencionada sob no 6834 , novamente ao Dr. White, que o identificou em nível genérico, enquadrando-o em Diphaulaca. Esse especialista ainda informou que os dois gêneros em questão, Diphaulaca e Chlamophora, possuem espécies bastante próximas. Posteriormente, exemplares das duas coletas foram examinados pelo Dr. Gerhard Scherer, Museum G. Frey, Alemanha, que os identificou como Diphaulaca viridipennis Clark, 1865.

$\mathrm{Na}$ literatura brasileira encontram-se referências de $D$. volkameriae (Fabr.) como praga de folhagem de leguminosas: feijão, Phaseolus vulgaris $L$. (MONTE, 1933, \& REGO, 1945), feijão-macassar, Vigna sinensis Endl. (CAVAL CANTE \& CAVALCANTE, 1975) e fava, Vicia fabae L (SCHLOTTFELD, 1944); há, todavia, a possibilidade de ter ocorrido erro de identificaçăo, podendo ser essa a espécie D. striata (BARBER, 1941). GUÉRIN (1953) cita a ocorrência no Brasil das espécies $D$. volkameriae, $D$. advena Fabr., $D$. costulata Clark, $D$. pallipes Jac., $D$. rubens Clark e $D$. striata Klug, não fazendo, contudo, referência à espécie $D$. viridipenniș.

(5) Os autores agradecem ao Dr. Sérgio Arce Gomez, pesquisador da EMBRAPA, UEPAE de Dourados, a doação dos insetos à Seção de Entomologia Fitotécnica do Instituto Agronómico. 
Com o objetivo de conhecer o comportamento de cultivares e linhagens de soja em relação ao dano foliar causado por adultos de $D$. viridipennis, conduziu-se o presente estudo.

\section{MATERIAL E MÉTODOS}

O germoplasma estudado compreende os cultivares IAC 8, de grande aceitação pelos agricultores do Estado de São Paulo, e Santa Rosa, testemunha suscetível a crisomelideos (LINK \& COSTA, 1978; REZENDE et alii, 1980; ROSSETTO \& NAGAI, 1980, e ROSSETTO et alii, 1981) e as linhagens PI 171451, pouco danificada por Colaspis sp. (ROSSETTO \& NAGAI, 1980), PI 227687, também resistente a Colaspis sp. (REZENDE et alii, 1980,e ROSSETTO \& NAGAl, 1980) e a D. speciosa (REZENDE et alii, 1980, e ROSSETTO et alii, 1981), PI 229358, PI 274453, IAC 78-2318, IAC 79-1823, IAC 80-1177, IAC 80-596-2 e D 72-9601-1. Todas essas linhagens apresentam resistência variável a uma ou mais espécies de insetos que se alimentam de soja e já foram estudadas em trabalhos de resistência de soja a insetos (LOURENÇĀO \& MIRANDA, 1983; LOURENÇĀO et alii, 19.95a, e LOURENÇĀO et alii, 1985b), com exceção da IAC 80-1177, material que, em experimentos preliminares, em nivel de campo, mostrou bom comportamento em relação a percevejos pentatomídeos.

Conduziram-se quatro experimentos, três tipo livre chance de escolha e um sem chance de escolha, em condições de casa de vegetação, no Centro Experimental de Campinas, a fim de avaliar o desempenho de materiais frente a $D$. viridipennis. Nos testes tipo livre chance de escolha, devido a restrições de número e tamanho das gaiolas, que comportavam apenas cinco vasos, os tratamentos a testar foram divididos em três experimentos distintos, conduzidos em épocas diferentes, sempre conservando o tratamento mais resistente e o mais suscetivel e incluindo outros três.

Para o primeiro experimento, a 17-11-83 foi feita semeadura dos cinco tratamentos (Quadro 1) em vasos de alumínio com capacidade para 1,5 litro, desbastando-se depois da geminação para duas plantas por vaso. Dezenove dias após o plantio, os vasos foram agrupados de acordo com o delineamento de blocos ao acaso, com seis repetiçōes. Cada parcela constituiu-se de um vaso com duas plantas e, cada bloco, de cínco vasos distribuídos em círculo sobre o balcão da casa de vegetação. A fim de evitar qualquer efeito da altura da planta sobre o comportamento do inseto, já que os tratamentos apresentam diferentes portes e intensidade de crescimento, nivelaram-se os vasos para que as plantas de um mesmo bloco ficassem com a mesma altura. A seguir, cada bloco foi coberto por uma gaiola cilíndrica de tela de náilon e nele liberados 40 adultos de $D$. viridipennis coletados em campo sobre soja IAC 77-530. A 12-12-83, sete dias após o confinamento dos insetos, avaliou-se o experimento, estimando-se visualmente a porcentagem de área foliar comida em todos os foliolos das duas plantas de cada parcela. 
Ainda a 12-12-83, procedeu-se ao plantio dos cinco materiais (Quadro 2) no segundo experimento, que foi conduzido de maneira idêntica ao primeiro. A infestação, realizada a 28-12-83, consistiu na liberação de 50 , em vez de 40 insetos por bloco, uma vez que se pretendeu aumentar o dano para facilitar a discriminação dos tratamentos, que foram avaliados também sete dias após a infestação.

Conduzido da mesma forma que os dois anteriores, o terceiro experimento teve seus materiais (Quadro 3) plantados a 18-1-84. A infestação de 50 insetos por gaiola foi realizada a 7-2-84 e, a avaliação, nove dias depois.

O quarto experimento constou de teste sem chance de escolha envolvendo o cultivar Santa Rosa e a linhagem PI 227687, que foram plantados a 5-1-84 nos vasos de alumínio já especificados, com desbaste para duas plantas após a germinação. Dezenove dias depois, os vasos foram agrupados em blocos ao acaso, com dez repetições, sendo cada vaso com duas plantas (parcela) envolvido por gaiola de tela de náilon, dentro da qual liberaram-se oito adultos de $D$. viridipennis. A 31-1-84, avaliou-se a área foliar comida, da mesma forma feita nos experimentos anteriores.

As porcentagens de área foliar comida foram transformadas em arco seno $\sqrt{\% / 100}$ e, as médias, comparadas pelo teste de Tukey ao nivel de $5 \%$.

\section{RESULTADOS E DISCUSSÃO}

No quadro 1 acham-se as médias de área foliar comida por $D$. viridipennis nas linhagens PI 227687, PI 229358 e Pl 171451 e nos cultivares IAC 8 e Santa Rosa; verifica-se o maior dano neste úitimo, que também apresenta suscetibilidade a outros crisomelídeos que atacam a soja: Colaspis sp. (REZENDE et alii, 1980, e ROSSETTO \& NAGAI, 1980), D. speciosa (LINK \& COSTA, 1978; REZENDE et alii, 1980, e ROSSETTO et alii, 1981), Andrector hybridus Bechiné (LINK \& COSTA, 1978) e $C$. arcuata (ROSSETTO et alii, 1981). A linhagem PI 227687, considerada resistente a Colaspis sp. (REZENDE et alii, 1980, e ROSSETTO \& NAGAl, 1980) e $D$. speciosa (REZENDE et alii, 1980, e ROSSETTO et alii, 1981), teve a menor perda de área foliar, diferindo significativamente dos demais tratamentos.

Considerando Pl 227687 e 'Santa Rosa' como, respectivamente, padrōes de resistência e suscetibilidade, e introduzindo no estudo as linhagens IAC 78-2318, IAC 79-1823 e IAC 80-1177, conduziu-se o segundo experimento, cujos resultados se encontram no quadro 2 . Observa-se maior variação de área foliar comida entre os tratamentos, possivelmente influenciada pelo maior número de insetos em relação ao primeiro experimento e/ou por fatores genéticos, que poderiam conferir diferentes graus de resistência. Novamente PI 227687 e 'Santa Rosa' sofreram, respectivamente, o menor e o maior desfolhamento. 
QUADRO 1. Médias de porcentagem de área foliar comida por Diphaulaca viridipennis em dois cultivares e três linhagens de soja em teste tipo livre chance de escolha. Campinas, 1983

\begin{tabular}{llc}
\hline Tratamento & \multicolumn{1}{c}{ Origem } & Área foliar comida* \\
& & $\%$ \\
& & $8 \mathrm{a}$ \\
PI 227687 & Okinawa, Japão & $14 \mathrm{~b}$ \\
PI 229358 & Tóquio, Japão & $15 \mathrm{~b}$ \\
PI 171451 & Japão & $17 \mathrm{~b}$ \\
'IAC 8' & 'Bragg' x E 70-51 & $19 \mathrm{~b}$ \\
'Santa Rosa' & D 49-772 X La 41-1219 & 28,8 \\
\hline C.V. \% & & \\
\hline
\end{tabular}

*Médias seguidas pela mesma letra não diferem significativamente entre si pelo teste de Tukey a $5 \%$.

QUADRO 2. Médias de porcentagem de área foliar comida por Diphaulaca viridipennis em um cultivar e quatro linhagens de soja em teste tipo livre chance de escolha. Campinas, 1983/84

\begin{tabular}{llc}
\hline Tratamento & \multicolumn{1}{c}{ Origem } & Área foliar comida* \\
\hline & & $\%$ \\
PI 227687 & Okinawa, Japão & $24 \mathrm{a}$ \\
IAC 78-2318 & D 72-9601 x IAC 73-227 & $33 \mathrm{~b}$ \\
IAC 79-1823 & IAC 70-308 x D 72-9601 & $38 \mathrm{~b}$ \\
IAC 80-1177 & D 72-9601-1 x ('Davis x PI 227687) & $40 \mathrm{bc}$ \\
'Santa Rosa' & D 49-772 x La 41-1219 & $47 \mathrm{c}$ \\
\hline C.V. \% & & 13,3 \\
\hline
\end{tabular}

* MÉdias seguidas pela mesma letra nāo diferem significativamente entre si pelo teste de Tukey a $5 \%$. 
Os resultados do terceiro experimento (Quadro 3) mostram que a PI 227687 foi a menos danificada por $D$. viridipennis, enquanto as perdas de área foliar apresentadas pelas linhagens PI 274453, IAC 80-596-2 e D 72-9601-1 não diferiram da testemunha suscetivel, o cultivar Santa Rosa. Apesar de ser o material mais resistente, $\mathrm{PI} 227687$ teve $42 \%$ de área foliar comida, dano relativamente alto, que pode ser explicado pelo periodo maior de infestação, nove dias, em que as plantas ficaram expostas ao ataque dos besouros.

QUADRO 3. Médias de porcentagem de área foliar comida por Diphaulaca viridipennis em um cultivar e quatro linhagens de soja em teste tipo livre chance de escolha. Campinas, 1984

\begin{tabular}{llc}
\hline Tratamento & \multicolumn{1}{c}{ Origem } & Área foliar comida* \\
\hline & & $\%$ \\
PI 227687 & Okinawa, Japão & $42 \mathrm{a}$ \\
PI 274453 & Okinawa, Japão & $53 \mathrm{~b}$ \\
IAC 80-596-2 & IAC 73-228 x 'UFV-1' & $59 \mathrm{~b}$ \\
D 72-9601-1 & D 66-8666 x ('Bragg' x PI 229358) & $61 \mathrm{~b}$ \\
'Santa Rosa' & D 49-772 x La 41-1219 & $61 \mathrm{~b}$ \\
\hline C.V. \% & & 10,5 \\
\hline
\end{tabular}

* Médias seguidas pela mesma letra não diferem significativamente entre si pelo teste de Tukey a $5 \%$.

Como a menor alimentação de um inseto em determinado material em teste tipo livre chance de escolha não representa necessariamente que a resistência observada seja efetiva, torna-se sempre desejável complementar o estudo com um teste de obrigatoriedade, ou seja, um teste em que o inseto não tenha chance de escolha entre esse e os demais materiais. No presente caso, utilizaram-se a PI 227687, que foi o material menos danificado, e o cultivar Santa Rosa, testemunha suscetivel. As médias do experimento - Quadro 4 - indicam comportamento semelhante dos dois tratamentos em relação ao dano causado pelo inseto; esses dados evidenciam que a resistência tipo não-preferência para alimentação apresentada pela linhagem PI 227687 nos três experimentos anteriores não se mantém sob condiçōes de obrigatoriedade de alimentação por adultos de $D$. viridipennis.

Portanto, dentro do germoplasma estudado, não se constatou nenhum material com perspectiva de ser utilizado para resistência $a$. viridipennis em um programa de melhoramento de soja a insetos. 
QUADRO 4. Médias de porcentagem de área foliar comida por Diphaulaca viridipennis em um cultivar e uma linhagem de soja em teste sem chance de escolha. Campinas, 1984

\begin{tabular}{lc}
\hline Tratamento & Área foliar comida* \\
\hline PI 227687 & $31 \mathrm{a}$ \\
'Santa Rosa' & $32 \mathrm{a}$ \\
\hline C.V.\% & 10,1 \\
\hline
\end{tabular}

* Médias seguidas pela mesma letra não diferem significativamente entre si pelo teste de Tukey a $5 \%$.

\section{SUMMARY}

\section{RESISTANCE OF SOYBEAN TO INSECTS. V. FEEDING PREFERENCE OF DIPHAULACA VIRIDIPENNIS CLARK, 1865, ADULTS AMONG SOYBEAN GENOTYPES}

The feeding preference of Diphaulaca viridipennis Clark, 1865 , (Coleoptera: Chrysomelidae) was studied in eleven soybean genotypes, in four experiments under greenhouse conditions at Campinas, State of São Paulo, Brazil, during the summer of 1983/84. Three free-choice-type experiments were carried out. In the first, the plant introduction PI 227687 was less defoliated by $D$. viridipennis than the other genotypes: 'IAC 8', PI 171451, PI 229358 and 'Santa Rosa'; the latter was the most damaged. In the second and third experiments, PI 227687 and 'Santa Rosa' were utilized as resistant and susceptible checks respectively; the other six genotypes studied (PI 274453, IAC 78-2318, IAC 79-1823, IAC 80-1177, IAC 80-596-2 and D 72-9601-1) presented intermediate performance between the checks. A non-choice-type experiment was set up with the two checks, PI 227687 and 'Santa Rosa'; the percentage of leaf area eaten in both treatments was similar, indicating that the PI 227687 resistance to $D$. viridipennis disappears when there is obligatoriness in feeding conditions.

Index terms: resistance to insects; feeding preference; soybean, Glycine max (L.) Merrill; Diphaulaca viridipennis Clark, 1865 ; leaf area eaten. 


\section{REFERÊNCIAS BIBLIOGRÁFICAS}

BARBER, H.S. Some fleabeetles injurious to beans in Tropical.America (genus Diphaula$c a$, family Chrysomelidae). Proceedings Entomological Society of Washington, 43(3):65-68, 1941.

CAVALCANTE, R.D. \& CAVALCANTE, L.C.C. Diphaulaca volkameriae (Fabr. 1742), praga do feijāo macassar (Vigna sinensis Endl.) no Ceará. Fitossanidade, Fortaleza, 1(3):98, 1975.

DEGASPARI, N. \& GOMEZ, S.A. Distribuiçāo geogrăfica e abundância estacional dos insetos pragas da soja e seus inimigos naturais, In: REUNIÄO DE PESQUISA DE SOJA - REGIÄO CENTRO, 3., Dourados, MS, 1979. p.182-185.

GUÉRIN, J. Coleópteros do Brasil. São Paulo, Faculdade de Filosofia, Ciências e Letras, USP, $1953.356 \mathrm{p}$.

LINK, D. \& COSTA, E.C. Danos causados por besouros crisomelídeos em soja. Revista do Centro de Ciências Rurais, Santa Maria, 8(3):245-250, 1978.

LOURENÇÃO, A.L. \& MIRANDA, M.A.C.de. Resistência de soja a insetos. I. Comportamento de linhagens e cultivares em relação a Epinotia aporema (Wals.) (Lepidoptera: Tortricidae). Bragantia, Campinas, 42:203-209, 1983.

; ROSSETTO, C.J. \& MIRANDA, M.A.C.de. Resistência de soja a insetos. III. Seleção de linhagens resistentes a percevejos. Bragantia, Campinas, 44(1):77-86, 1985a.

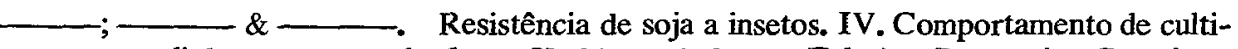
vares e linhagens em relaçáo a Hedilepta indicata (Fabr.). Bragantia, Campinas, 44(1):149-157, $1985 \mathrm{~b}$.

MASSARIOL, A.A.; RAMIRO, Z.A. \& CALCAGNOLO, G. Insetos observados na cultura da soja no Estado de São Paulo. O Biologico, São Paulo, 45:83-87, 1979.

MONTE, O. A vaquinha-azul do feijão. Boletim de Agricultura, Zootecnia e Veterinária, Belo Horizonte, 6(1):49-50, 1933.

REGO, C. do V.; GOMES, J.G. \& ALVIM, G.B. Doenças e pragas das plantas de horta. Rio de Janeiro, Ministério da Agricultura, Serviço de Documentação, 1945. 230p.

REZENDE, J.A.M.; ROSSETTO, C.J. \& MIRANDA, M.A.C.de. Comportamento de populações paternais e F1 de soja em relação a Colaspis sp. e Diabrotica speciosa (Germar, 1824). Bragantia, Campinas, 39:15-20, 1980.

RODINI, E.S.O. \& GRAZIA, J. Abundância de algumas espécies de insetos (Coleoptera e Hemiptera) em soja (Glycine max (L.) Merrill) no município de Aguaí, SP. In: SEMINÁRIO NACIONAL DE PESQUISA DE SOJA, 1., Londrina, 1979. Anais. v.2, p.17-22.

ROSSETTO, C.J.; NAGAI, V.; IGUE, T.; ROSSETTO, D. \& MIRANDA, M.A.C.de. Preferência de alimentação de adultos de Diabrotica speciosa (Germar) e Cerotoma arcuata (Oliv.) em variedades de soja. Bragantia, Campinas, 40:179-183, 1981.

ROSSETTO, D. \& NAGAI, V. Ensaio de livre escolha para o coleóptero Colaspis sp. em seis variedades de soja. Bragantia, Campinas, 39:195-198, 1980.

SCHLOTTFELDT, C.S. Insetos encontrados em plantas cultivadas e comuns - Viçosa, Minas Gerais. Revista Ceres, Viçosa, 6(31):52-65, 1944.

WILLIAMS, R.N.; PANAIA, J.R.; MOSCARDI, F.; SICHMANN, W.; ALLEN, G.E.; GREENE, G. \& LASCA, D.H.C. Principais pragas da soja no Estado de São Paulo. São Paulo, Secretaria da Agricultura, CATI-DOT-DF, 1973. 18p. 